\title{
Recomendação de Conteúdo integrada à Escrita Coletiva Digital: qualificando processos e ampliando possibilidades
}

\author{
Sandra Andrea Assumpção Maria - sandradeia@ gmail.com - PPGIE/UFRGS \\ Alexandra Lorandi Macedo - alorandimacedo@ gmail.com - SEAD/UFRGS \\ Patricia Alejandra Behar - pbehar@ terra.com.br-PPGIE/UFRGS
}

\begin{abstract}
Resumo: Pesquisar materiais que sirvam de apoio à Escrita Coletiva Digital, independente do tema em desenvolvimento, é um desafio que tem se intensificado a cada dia. O volume de informações na Internet cresce numa velocidade avassaladora e, aliado a isso, estão os mais diferentes estímulos que competem com a necessidade da manutenção de foco e atenção dos autores. Pensando nisso é que foi implementada, dentro do Editor de Texto Coletivo (ETC), uma ferramenta de Recomendação de Conteúdo (RecETC) que, com base na escrita dos autores, indica materiais em formato de texto, imagem e vídeo. Sua aplicação ocorreu em 3 turmas (Graduação, PósGraduação e Extensão) e teve positivo impacto tanto para alunos, no auxílio à produção textual, quanto para professores, quando da mediação desta produção.
\end{abstract}

Palavras-chave: Escrita Coletiva Digital, Recomendação de Conteúdo, Editor de Texto Coletivo

\section{Integrated Content recommendation to Digital Collective Writing: qualifying processes and expanding possibilities}

\begin{abstract}
Search for materials that support digital writing, regardless of the theme under development, is a challenge that has intensified every day. The volume of information on the Internet is growing at an overwhelming speed, allied to it are the most different stimulus that compete with the need to focus attention and maintenance of the authors. Thinking about it, a Content Recommendation tool (RecETC) was implemented within the Collective Text Editor (ETC), which, based on the authors' writing, indicates text, image and video format materials. Its application occurred in 3 classes (Undergraduate, Graduate and Extension) and had a positive impact both for students, in aid to textual production, and for teachers, when mediation of this production.
\end{abstract}

\section{Keywords: Collective Digital Writing, Content Recommendation, Collective Text Editor}

\section{Introdução}

Este estudo tem por objetivo analisar o impacto do RecETC, que é um Recomendador de Conteúdo criado para o Editor de Texto Coletivo (ETC) ${ }^{1}$, no desenvolvimento das produções textuais coletivas. A aplicação e a análise ocorreram em 3 turmas (Graduação, Pós-Graduação e Extensão) no qual experienciaram encontros presenciais e a distância, de acordo com os propósitos educacionais de cada atividade. O intuito, quando do planejamento desta ferramenta, foi sanar uma necessidade emergente identificada em situações reais que envolvem a Escrita Coletiva Digital (ECD).

$\mathrm{O}$ volume de dados disponível na Internet tem causado impactos positivos e negativos para os usuários que realizam pesquisas. De um lado, a Internet permite disponibilizar diversos tipos de conteúdos e formatos de materiais, ampliando as 
possibilidades de consulta. Do outro, tem dificultado o encontro dos mesmos, tendo em vista a grande quantidade de informações e, muitas vezes, disponibilizada sem qualquer cuidado com os dados e descrições que possam melhor identificar o referido material. Logo, buscar conteúdo que sirva de referência para uma produção textual coletiva tornase um desafio tanto para alunos quanto para professores.

A busca por materiais relevantes no âmbito da Internet, pode implicar em resultados que se distanciam do foco da escrita, tendo em vista os seguintes aspectos: o volume de dados apresentados, a forma como eles são disponibilizados - sem cuidados com descrições adequadas que possam identifica-los, e como são procurados pelo usuário. A expressiva carga de informações disponíveis pode ocasionar distrações, direcionando o usuário para outros assuntos que não estão relacionados ao texto que está sendo produzido. A disponibilização em massa, impulsionada pelas tecnologias da Web 2.0, ao mesmo tempo que amplia a diversidade de materiais, carece de padronização e informação adequada no que tange ao processo de descrição e identificação dos conteúdos. Do mesmo modo, as palavras-chave utilizadas para disparar a busca podem não representar uma boa consulta, pois os usuários podem ter dificuldades para descrever ou detalhar seus interesses. Foi diante dessa necessidade que, com base nos estudos de Sistemas de Recomendação (SR), desenvolveu-se uma ferramenta capaz de minerar e recomendar diferentes formatos de materiais aos autores participantes de uma produção coletiva. Será sobre essa ferramenta, e tendo como eixo a ECD, que desenvolver-se-á este estudo. Para isso, a próxima seção define como, aqui, entende-se o conceito de ECD, segue com a apresentação da ferramenta RecETC, detalha os aspectos que motivaram o seu desenvolvimento e, por fim, apresenta e analisa os dados coletados a partir de situações práticas.

\section{Escrita Coletiva Digital}

$\mathrm{O}$ avanço das tecnologias digitais tem produzido impactos em diferentes áreas e, uma das que tem mostrado significativas conquistas, é a da educação. São inúmeras as possibilidades de utilizar recursos digitais como apoio ao processo de ensino e aprendizagem e, nesse contexto, as ferramentas que favorecem o desenvolvimento de atividades coletivas têm marcado seu protagonismo. Isso porque as práticas apoiadas nas trocas interindividuais têm mostrado significativo potencial frente à construção do conhecimento.

Nesse cenário, as relações colaborativas e/ou cooperativas estão se destacando como um meio para promover a construção coletiva de saberes, considerando a complexidade e as características que envolvem esse movimento dinâmico. Desse movimento, faz parte a Escrita Coletiva Digital (ECD) que é uma estratégia que está sendo utilizada para se referir a construção de um texto por mais de um autor, onde sugere-se uma organização prévia e constante dos envolvidos.

Behar et al. (2004) define texto coletivo como uma rede de saberes em movimento, visto que está em constante alteração. Ele produz e é produzido por meio dos múltiplos saberes, das múltiplas culturas entrelaçadas. Logo, é "bordado" pelos vários autores que o constituem, manifestando-se de maneira aberta e dinâmica na coletividade.

A produção textual coletiva pode acontecer tanto no contexto presencial como no digital e costuma ser vista como uma atividade complexa, já que requer articulação, empenho e comprometimento de todos os autores. Ao optar pela utilização dessa estratégia, tanto o professor quanto os alunos, precisam compreender as especificidades que envolvem a sua realização. De um lado, os alunos precisam saber quais são suas 
atribuições frente ao desafio e também precisam entender a dinâmica deste processo. De outro, os professores necessitam construir um planejamento que detalhe como será o desenvolvimento dessa atividade. De modo geral, devem considerar: aspectos organizacionais de sua aplicação, as particularidades da tecnologia a ser utilizada, o acompanhamento e auxílio que deverão ser prestados aos alunos.

Segundo Maria (2016), as principais características que envolvem a atividade de ECD são: as possibilidades de uma escrita hipertextual, o uso de recursos tecnológicos próprios para desenvolvimento e acompanhamento, a flexibilidade do tempo e do período para edição, o uso de diferentes estratégias pedagógicas para a sua aplicação, as relações colaborativas e/ou cooperativas que podem ser estabelecidas, a interação e interatividade realizada pelos sujeitos/tecnologia, o trabalho em equipe e o exercício da autoria, o respeito pelo outro, a compreensão de interdependência e os níveis de negociação entre a equipe.

No que se refere a escrita hipertextual, os autores possuem a liberdade para incorporar ao texto diferentes recursos multimídia, assim como possibilitar que o leitor obtenha mais informações sobre a escrita ao fazer uso de hiperlinks. Dessa forma, a produção que está sendo realizada pelos autores é enriquecida a medida que o texto é conectado com outras fontes, favorecendo uma leitura mais dinâmica, não linear e aprofundada do texto.

Outro aspecto relacionado à ECD está na disponibilização e utilização de recursos tecnológicos específicos para apoiar os autores no desenvolvimento do texto e no acompanhamento, seja por parte dos próprios autores ou por professores. Funcionalidades como uso de dicionários, correção ortográfica e gramatical, ferramentas de formatação, registro de versões do texto, uso de Sistemas de Recomendação, recursos que favoreçam a interação e a comunicação são exemplos de mecanismos que podem facilitar a produção textual no contexto digital. Com a evolução da web, muitos recursos antes utilizados de forma off-line foram integrados aos atuais editores, assim como outros foram construídos com o propósito de oferecer recursos e mecanismos cada vez mais personalizados, visando atender diferentes necessidades. Diante disso, algumas características da Educação a Distância (EaD) também podem ser percebidas na atividade de ECD. Acessos síncronos ou assíncronos a partir de diferentes espaços geográficos são exemplos de possibilidades que favorecem melhor articulação para estudos relacionados e interação entre os autores.

No que tange aos aspectos pedagógicos, o professor possui a liberdade para definir limitações e alternativas, como: a seleção dos recursos multimídia, as condições para favorecer e fomentar a colaboração e/ou cooperação, as dinâmicas para formação de grupos, entre outros. Além disso, o professor também pode participar, de forma dinâmica, do processo de escrita e não apenas na avaliação do texto como produto final. A oportunidade de acompanhamento realizada pelo professor está diretamente relacionada aos objetivos pedagógicos definidos pelo mesmo. No entanto, a transparência favorecida pelas tecnologias ao longo da construção do texto pode auxiliar na mediação pedagógica e potencializar a interação entre os envolvidos.

O trabalho em equipe é um dos pontos-chave do processo de ECD. Para se ter sucesso nesta ação, é necessário que os autores tenham clareza em relação às suas atribuições e estabeleçam uma constante comunicação, pois a escrita coletiva depende da interação entre os membros e do estabelecimento de níveis de consenso para alcançar seus objetivos. Além disso, respeitar a opinião e sugestões do outro é ter compreensão da necessidade de interdependência entre os envolvidos visando um objetivo comum.

Nesse sentido, este estudo apoiou-se nos conceitos aqui destacados e na observação dos sujeitos ao longo de ECD para testar suas hipóteses e construir seu 
próprio olhar sobre os processos oriundos desse movimento. Para viabilizar o estudo, utilizou-se o Editor de Texto Coletivo (ETC) que é um editor online $e$ deu-se foco ao uso de um Sistema de Recomendação, apresentados na próxima seção.

\section{A integração de um Sistema de Recomendação em um Editor de Texto Coletivo}

O Editor de Texto Coletivo (ETC) vem sendo aprimorado a partir de depoimentos e avaliações de professores e alunos usuários do sistema (MACEDO et al.,2010). A partir de situações de ensino e de aprendizagem no ETC, identificou-se a necessidade de desenvolver uma ferramenta de apoio à Escrita Coletiva Digital (ECD). O objetivo foi integrar, ao Editor, um recurso que, com base na produção textual, retornasse diferentes formatos de materiais que pudessem servir de auxílio à produção escrita. Assim, foi desenvolvido e implementado, no ETC, o RecETC (Recomendador do ETC). Nele, a recomendação é realizada com base no conteúdo desenvolvido pelos autores através da identificação dos termos mais frequentes e a relação destes com os materiais armazenados em uma base de dados. A figura 1 ilustra o RecETC e seus recursos.

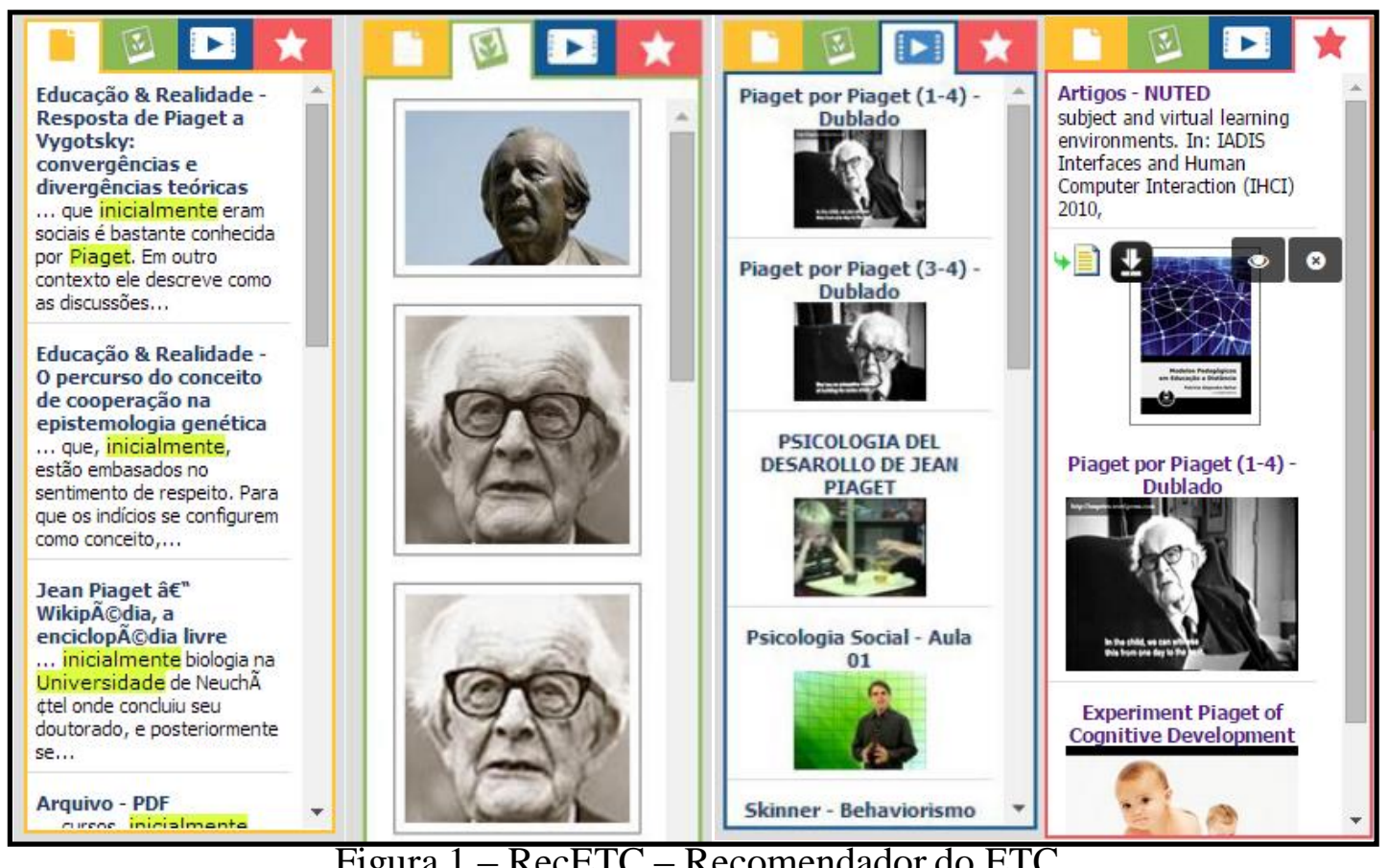

Figura 1 - RecETC - Recomendador do ETC

O funcionamento do RecETC apoia-se em três subsistemas: o indexador, o minerador de texto e o sistema de consultas. $O$ indexador tem a função de salvar o endereço de páginas web que contenham os diferentes tipos de materiais (imagens, textos e vídeos) a fim de alimentar o banco de dados do Recomendador. A partir de um único registro o sistema é capaz de buscar uma página "P", guardar seu conteúdo e fazer o mesmo processo com cada página referenciada pelos links deste primeiro endereço indexado. O segundo subsistema, que dá sequência às ações da ferramenta, é o minerador. Com base na técnica de Mineração de Textos, ele extrai os termos mais frequentes da produção textual identificando o assunto que está sendo tratado. Além desta técnica, o usuário também pode cadastrar novas palavras-chave ou remover as que não achar pertinente a fim de compor os termos de busca. Chega, então, a vez do último subsistema, o de Consultas que, com base nas palavras-chave filtradas na etapa anterior, realiza três consultas. A primeira faz uma busca por textos (sites/artigos), a segunda por 
imagem e a terceira por vídeos (Youtube). Tais buscas são realizadas no banco de dados do próprio ETC que, por sua vez, é alimentado com páginas/sites pelo subsistema Indexador. A ordem de apresentação dos materiais é feita com base na quantidade de ocorrências das palavras-chave dentro do endereço do site/artigo, imagem ou vídeo. Além disso, também são priorizados resultados que contenham todas as palavras-chave mineradas, por exemplo: "sistema de recomendação", os resultados que contiverem tanto "sistema" quanto "recomendação" terão prioridade na busca.

Por fim, destaca-se que o RecETC tem a área de Favoritos, cujo objetivo é guardar todos os materiais marcados pelos usuários nas abas texto, imagens e vídeos. Essa área pode reservar materiais que sejam considerados, pelo grupo, como de maior relevância para apoiar a escrita ou como recomendação de leitura. Por ser este um espaço coletivo, os materiais marcados neste espaço são comuns a todos os integrantes.

Com o entendimento de como o RecETC funciona, este estudo segue com os fatos que motivaram a construção desta ferramenta, bem como com a análise dos dados coletados a partir de situações reais de aprendizagem.

\section{Impactos do RecETC sobre a produção textual e a prática pedagógica}

Há muito tem-se falado sobre o alto volume de informações que são geradas diariamente na Internet e, quando se trata de consulta acadêmica, a rede mundial de computadores já é a mais acessada fonte de pesquisa. No entanto, a dificuldade em gerenciar, selecionar e avaliar as informações alí disponibilizadas cresce na mesma proporção que o volume de novas informações diárias. Uma simples consulta na rede pode custar um valioso e imensurável tempo para seleção e eleição de conteúdos pertinentes e de aderência à área de interesse. Além da dificuldade crescente de selecionar as fontes de referência, quando a pesquisa é feita na web, a possibilidade de perder o foco da ação diante da imensa oferta e de diferentes estímulos é bastante significativa. Nesse sentido, justifica-se a implementação do RecETC no Editor de Texto Coletivo (ETC). Mas, a questão que se destaca aqui é: como o RecETC pode impactar o desenvolvimento das produções textuais coletivas?

Este estudo se propõe a analisar a questão feita sob duas diferentes perspectivas: a do aluno e a do professor. Na perspectiva do aluno, um dos relevantes aspectos que podem ser considerados é a relação que cada sujeito estabelece com as referências sugeridas pelo RecETC. Tal relação baseia-se nos valores de cada um, de onde derivam situações de equilíbrio ou desequilíbrio, uma vez que o grau de importância atribuído aos materiais recomendados poderá ser diferente para cada sujeito. Quando um conteúdo for recomendado por algum colega do grupo, mesmo que não tenha sido valorizado pelo sujeito que o recebeu em uma primeira leitura, este poderá ter um impacto diferente do primeiro contato dependendo da relação que cada sujeito estabelece com o colega. Nessa troca o respeito mútuo ou unilateral estão diretamente relacionados, onde no unilateral está presente a imposição feita de um sujeito para com o outro e o mútuo onde existe uma escala comum de valores e não há diferente nível hierárquico entre os envolvidos (PIAGET, 1973). Este movimento de valorização, ou não, de materiais recomendados pelo RecETC, avaliados e novamente recomendados pelos colegas é dinâmico e acontece de forma permanente durante o processo de escrita coletiva. O maior desafio é manter o equilíbrio nas trocas para que os esforços se debrucem mais sobre a escrita e menos nas regulações entre os pares. A vantagem que o RecETC apresenta nessa relação é que o conteúdo recomendado, por ter sofrido uma avaliação na filtragem da ferramenta, tem maior probabilidade de ser valorizado pelos 
sujeitos minimizando, assim, o primeiro movimento de consideração ou não das fontes recomendadas.

O RecETC recomenda três de formatos de conteúdo. Essa variedade pode impactar, de forma positiva, o resultado da escrita. Nem sempre o sujeito tem tempo suficiente e também paciência para procurar por diferentes formatos de conteúdo sobre um mesmo tema. Essa diversidade, além de ampliar a qualidade da consulta, também pode potencializar as relações que o sujeito estabelece com o conhecimento já construído por ele. Além disso, ter a oportunidade de analisar um conjunto de diferentes pontos de vista, pode ampliar o escopo argumentativo e contribuir para qualificar a escrita coletiva.

Se de um lado o RecETC pode favorecer os processos de escrita coletiva dos autores; de outro, poderá contribuir para também qualificar a prática pedagógica dos professores.

A partir da análise dos textos produzidos pelos alunos, se o professor identificar alguma necessidade de aprofundamento ou aprimoramento na escrita, ele poderá marcar como favorito fontes retornadas pelo RecETC. A valorização do professor pode dar um peso maior ao material recomendado pela ferramenta e, por vezes, poderá fazer sentido para o aluno. A valorização, ou não, feita pelo usuário ao material recomendado e/ou avaliado pelo professor, poderá ser conferida ao longo da produção textual, nas referências ou até nas discussões do grupo. Mas, se os usuários ignorarem as referências dirigidas pelo professor através do RecETC cabe investigar o nível de conhecimento destes sujeitos em relação ao tema trabalhado. Isso porque a falta ou limitação do conhecimento sobre o assunto pode não favorecer o estabelecimento de relações entre os sujeitos e o material recomendado, comprometendo, assim, a qualidade da produção textual (MACEDO, et al. 2012).

$\mathrm{O}$ fato de o recomendador retornar ao usuário uma significativa variedade de formatos de materiais; amplia, também para o professor, o universo da pesquisa, das relações e novas possibilidades de fontes que podem ser sugeridas, instigadas e proporcionadas aos alunos podendo potencializar as relações que os sujeitos estabelecem entre os conteúdos e a elaboração do novo.

Deste movimento, o professor pode identificar necessidades que sejam semelhantes, complementares ou divergentes entre os grupos e, sempre que for oportuno, poderá colocá-los em contato, compartilhando os materiais de referência (criando uma base única) e favorecendo, assim, o trabalho cooperativo em prol da qualificação da ECD.

\section{A coleta de dados e os resultados preliminares}

O RecETC foi utilizado e analisado em 3 turmas, a saber: Graduação (Pedagogia), PósGraduação (Educação e Informática na Educação - PPGEDU e PPGIE) e Extensão Universitária (Formação de Professores), da Universidade Federal do Rio Grande do Sul. O tema central dos cursos variou conforme sua natureza e objetivos específicos de suas aplicações. O ponto comum foi o desenvolvimento da Escrita Coletiva Digital (ECD), tendo como ferramenta de apoio o RecETC. O número total de participantes foi de 60 e o período de coleta dos dados deste estudo aconteceu entre o segundo semestre de 2015 e o primeiro semestre de 2016.

Para alcançar os objetivos, aplicou-se um questionário online e os alunos foram convidados a respondê-lo. Esse abrangeu questões objetivas e dissertativas, a fim de analisar o entendimento dos participantes em relação a dinâmica da atividade de ECD e do funcionamento do RecETC (questões técnicas e pedagógicas). No entanto, este 
estudo apoiou-se em dois objetivos centrais: (i) conhecer as impressões dos usuários sobre a ferramenta RecETC e (ii) identificar possível impacto da ferramenta na construção da ECD.

$\mathrm{O}$ acesso ao formulário não foi de caráter obrigatório e, por isso, alguns feedbacks foram dados em conversas informais entre alunos e professor (pesquisador). Em paralelo, os professores (pesquisadores) estudaram, acompanharam e mediaram a construção coletiva de textos no ETC com apoio do RecETC.

Os excertos sinalizados na tabela 1 e 2 foram destacados de questionários respondidos pelos participantes. Neles, nota-se que a percepção dos usuários remete à facilidade para realização da pesquisa e para seleção de conteúdos relacionados ao tema.

Tabela 1 - Percepção do usuário sobre o RecETC

Curso 1 - Extensão Universitária

Pergunta realizada: Como você percebeu o uso de um Sistema de Recomendação no contexto da escrita coletiva?

\section{Aluno 1:}

$\mathrm{R}$ : Importantíssimo, pois direciona a pesquisa para a construção.

\section{Aluno 2:}

R: O sistema de recomendação serve como uma filtragem que automaticamente reconhecem e categorizam as informações, onde facilita ao usuário a busca dos conteúdos específicos.

\section{Aluno 3:}

$\mathrm{R}$ : Os sistemas de recomendação nos auxiliam no filtro de informações, procurando indicar o que possa nos interessar. Assim nos facilitando na busca de conteúdos específicos.

\section{Aluno 4:}

$\mathrm{Na}$ pesquisa a filtragem é bastante difícil em textos, creio que o sistema de recomendação funciona como que forma automática reconhecendo e categorizando as informações, o que facilita ao usuário a busca dos conteúdos específicos.

Curso 2 - Graduação

\section{Aluno 1:}

R: A possibilidade de utilização dos textos indicados, baseados nas palavras-chave, facilita o acesso a outros materiais de qualidade para pesquisa e composiçãa da escrita. Fiz a experiência de procurar alguns textos, somente com a indicação do sistema de recomendação, e achei informações relevantes para a construção do meu texto. Para quem trabalha com Educação a Distância a ferramenta pode contribuir muito, para pesquisa de estudantes, que muitas vezes utilizam a internet, mas não obtém materiais de qualidade e de cunho científico para suas pesquisas.

Aluno 2:

R: Interessante e motivador. Este tipo de sistema tem muito a somar para análise e entendimento de contextos, principalmente os mais complexos.

Curso 3 - Pós-Graduação 


\section{Aluno 1:}

R: Foi fácil utilizar e os textos indicados foram muito bons, utilizei eles como base para minha produção.

Aluno 2:

$\mathrm{R}$ : A ferramenta apresentou vídeos relevantes e de acordo com o tema que estávamos escrevendo. Foi legal ver o material ser indicado. Eu teria ido buscar só textos como base, ter visto $o$ vídeo foi um plus.

\section{Aluno3:}

$\mathrm{R}$ : A ferramenta foi bastante útil. Os textos que foram recomendados foram comentados pelo grupo. Ajudou o grupo a dar foco, foi o fio condutor para dar direção para o nosso trabalho. Facilitou a escrita.

Quando questionados sobre a percepção que tiveram do RecETC, os usuários destacaram que a ferramenta facilitou a pesquisa e o acesso ao conteúdo, além de ajudar a direcionar a escrita.

Oferecer conteúdo relacionado à produção textual sem exigir que o usuário construa todo o percurso da pesquisa, pode auxiliar na qualificação do texto, bem como auxiliar nas trocas interindividuais. Sabe-se que o desenvolvimento de uma pesquisa requer: seleção, julgamento, estudo e compartilhamento das impressões sobre as fontes consultadas. Esse processo, por si, já é um grande desafio. Somado a isso, está a tarefa de negociar diferentes pontos de vista entre os integrantes de um grupo, uma vez que a interpretação dos materiais é feita a partir de diferentes perspectivas. Neste cenário, pode-se inferir que o fato de o RecETC filtrar as fontes de consulta, pode minimizar possíveis ruídos entre os usuários e a dificuldade quando da manutenção da clareza e foco enquanto fazem suas buscas em sites abertos. Exemplo disso está quando o Aluno 3 do Curso de Pós-Graduação diz que os resultados do Recomendador ajudaram a dar foco na escrita, que foi o fio condutor para o trabalho e que facilitou a produção textual.

Tabela 2 - Percepção do usuário sobre o RecETC na produção textual

\section{Curso 1 - Extensão Universitária \\ Pergunta realizada: O RecETC colaborou no processo de produção textual? Explique como foi a experiência de utilização dessa funcionalidade.}

\section{Aluno 1:}

R: O RecETC é um bom mecanismo, pois permite a busca através de palavras-chave, tanto artigos, textos, vídeos e figuras, dando suporte para a construção do texto através da quantidade, qualidade e diversidade de informações.

\section{Aluno 2:}

R: O RecETC colaborou no meu processo de aprendizagem diante da produção textual, pois consegui obter informações através de artigos e textos. As imagens e vídeos não me deram suporte, pois não consegui visualizar.

\section{Aluno 3:}

R: O RecETC foi útil porque o grupo não perdeu tempo com a pesquisa já que o material já vinha indicado na tela. Nós conversamos direto sobre o que estava indicado e usamos os textos e artigos como referência. 


\begin{abstract}
Aluno 4:
R: Não creio que tenha ajudado muito, pois esperava que me mostrasse artigos disponíveis na web, por exemplo. O sistema retornou pouca coisa sobre isso. Quando já tínhamos terminado, fiquei me perguntando se isso não ocorreu pela pouca progressão que o texto teve, acredito que tenha contribuído, mesmo que eu tivesse inserido e também excluído algumas palavras-chave.
\end{abstract}

\title{
Curso 2 - Graduação
}

\section{Aluno 1:}

R: O material que o RecETC apresentou me ajudou a entender melhor o assunto.

\section{Aluno 2:}

R: Colaborou porque a ferramenta é ágil e prática na busca de informações. Ele recomendou textos e artigos que estavam de acordo com o que estava sendo escrito pelo grupo.

\section{Curso 3 - Pós-Graduação}

\section{Aluno 1:}

R: A ferramenta possibilitou uma nova forma de trabalho dentro da construção de texto. $\mathrm{Eu}$ e o grupo conversamos sobre os textos recomendados e também sobre os vídeos. Ampliou a pesquisa que eu faria, porque eu teria concentrado somente em textos acadêmicos.

\section{Aluno 2:}

R: Foi legal poder discutir direto sobre os textos recomendados porque todos já sabiam do que se estava falando. Isso tornou a discussão mais centrada, mais objetiva. Não ampliamos muito o leque, fomos direto ao ponto, não perdemos o foco como já aconteceu em outras vezes.

No que tange à identificação de possível impacto do RecETC na ECD, nota-se que a ferramenta funcionou, principalmente, como suporte para pesquisa, como foco para as discussões coletivas, como fonte alternativa de materiais (destacado pelo Aluno 1 do curso de Pós-Graduação), oferecendo quantidade, qualidade e diversidade de referências sobre os temas trabalhados. Nesse sentido, entende-se que, direta ou indiretamente, a ferramenta impactou a produção textual, ora como referência teórica, ora auxiliando o grupo a manter o foco da pesquisa.

Não menos importante que as demais, foi a colocação do Aluno 4 do curso de Extensão Universitária, que apontou como limitada a amostragem do RecETC. Isso pode ter acontecido em situações semelhantes e está sendo considerado na pesquisa como meta de análise para aprimoramento. O próprio aluno apontou como hipótese, que sua produção pode não ter sido suficiente para alimentar a base de recomendação do conteúdo. $\mathrm{O}$ estudo está considerando o exposto e indo além, a fim de qualificar a ferramenta para que a mesma retorne uma variedade cada vez maior de conteúdo.

\section{Conclusões}

Este estudo apresentou o Sistema de Recomendação denominado RecETC integrado ao Editor de Texto Coletivo (ETC). A motivação da pesquisa partiu da dificuldade, cada vez maior, de desenvolver pesquisas na Internet com o intuito de apoiar a Escrita 
Coletiva Digital (ECD). Sair da tela do Editor de Texto para buscar material de apoio está sendo um desafio. Isso porque encontrar o que, de fato, precisamos requer dedicação, paciência, seleção, análise, síntese e tempo. Aliado a isso, está também o desafio de manter o foco na pesquisa. São inúmeras as opções de consulta e de estímulos quando estamos navegando na rede. Para atender a esta necessidade é que foi pensado o RecETC que, sem precisar sair do ETC e com base na produção textual desenvolvida pelos usuários, recomenda textos, imagens e vídeos. A ideia é oferecer apoio com materiais de referência em diferentes formatos e minimizar as chances de perda de foco do usuário.

Com a implementação da ferramenta, teve-se o interesse em investigar, neste momento, a impressão dos usuários sobre o RecETC e se o mesmo impactou, de alguma forma, a ECD. As primeiras impressões dos usuários são positivas, pois entendem que o recurso oferece apoio para a produção textual, facilitam a pesquisa e favorecem na manutenção do foco da discussão e da escrita. Destaca-se que esta foi uma amostragem preliminar, mas que os dados coletados podem ser inspiradores para continuar investindo e aprimorando a ferramenta em busca de melhores patamares qualitativos em futuras aplicações práticas. Ademais, tem-se interesse em investir em estratégias pedagógicas que inspirem e qualifiquem novas ações educacionais.

\section{Notas de Texto}

${ }^{1}$ Editor de Texto Coletivo (ETC) - desenvolvido pelo Núcleo de Tecnologia Digital aplicada à Educação da Universidade Federal do Rio Grande do Sul (NUTED/UFRGS). Disponível em: <http://www.nuted.ufrgs.br/etc>.

\section{Referências}

BEHAR, P. A., et al. ETC: uma proposta de editor de texto coletivo na web. VII Congresso IberoAmericano de Informática Educativa. P. 363-373, 2004. Disponível em: http://www.ufrgs.br/limc/escritacoletiva/pdf/etc.pdf.

MARIA, S. A. RecETC: uma funcionalidade baseada na Recomendação de conteúdo para auxiliar no processo da Escrita Coletiva Digital. Porto Alegre, 2016. Proposta de Tese (Doutorado em Informática na Educação) - Programa de PósGraduação em Informática na Educação. Universidade Federal do Rio Grande do Sul. Porto Alegre, 2016.

MACEDO, A. L.; ZANK, C. ; BEHAR, P. A. ; VINADE, F. ETC: o que mudou e por quê. RENOTE. Revista Novas Tecnologias na Educação, v. 8, p. 10662, 2010.

MACEDO, A. L.; BEHAR, P. A.; REATEGUI, E. B. Acompanhamento da escrita coletiva a distância: tecnologia para apoiar a ação docente. RENOTE. Revista Novas Tecnologias na Educação, v. 10, p. 34, 2012.

PIAGET, Jean. Estudo Sociológicos. Rio de Janeiro. Forense, 1973. 